\title{
What Do Brazilian Pediatricians Know About Celiac Disease?
}

\author{
Camilo Vieira - Marília Matos - Thaise Quaresma • \\ Juliana de Oliveira • Cibele Dantas Ferreira • \\ Adriza Silva • Daniel Diniz-Santos • Luciana R. Silva
}

Received: 3 April 2010/Accepted: 29 June 2010/Published online: 15 July 2010

(C) Springer Science+Business Media, LLC 2010

\begin{abstract}
Introduction Celiac disease (CD) is a common illness, affecting $0.5-1 \%$ of the population. Its classic presentation consists of gastrointestinal symptoms, however, many extra-intestinal symptoms and some associated diseases have been studied. Pediatricians should know the typical and atypical presentations of CD and how to diagnosis and treat its complications. The aim of this study was describe what pediatricians in Brazil know about CD.

Methods A descriptive, cross-sectional study with pediatricians who participated in the Nestlé Pediatrics Course, in the city of Natal, Brazil, in 2008. They were asked to complete a self-applicable questionnaire covering aspects concerning the prevalence, diagnosis, and treatment of CD and the conditions associated with this disease.

Results A total of 632 pediatricians completed the questionnaire. The majority of respondents (82.9\%) were female. All the geographical regions of Brazil were
\end{abstract}

\section{Vieira}

Department of Pediatric Neurology, School of Medicine,

Federal University of Bahia, Salvador, Bahia, Brazil

M. Matos · T. Quaresma · J. de Oliveira

School of Medicine, Escola Bahiana de Medicina e Saúde Pública, Salvador, Bahia, Brazil

C. D. Ferreira · A. Silva · D. Diniz-Santos · L. R. Silva The Departments of Pediatric Neurology and Pediatric Gastroenterology and Hepatology of the Professor Edgard Santos Teaching Hospital and the Professor Hosannah de Oliveira Pediatrics Center, Federal University of Bahia, Salvador, Bahia, Brazil

\section{Vieira $(\bowtie)$}

Rua do Timbó 623, APT 802, Caminho das Árvores, 41820-660 Salvador, Bahia, Brazil

e-mail: camilovieira@uol.com.br represented. More than $65 \%$ of respondents had undergone specialist training in pediatrics, and $40 \%$ of respondents had worked as pediatricians for more than 25 years. Only $22 \%$ replied that celiac disease may be asymptomatic, $57 \%$ stated that antigliadin antibody measurement represents the best screening tool for the disease, and two-thirds replied that bowel biopsy would be the most indicated method. The pathologies and conditions associated with celiac disease were identified by fewer than $50 \%$ of respondents. Exclusion of gluten from the patient's diet was mentioned as the treatment for celiac disease by $86.4 \%$ of the pediatricians.

Conclusions We conclude that pediatricians have superficial information about $\mathrm{CD}$. The need for relevant information on celiac disease is fundamental and is recognized by the pediatricians themselves.

Keywords Celiac disease $\cdot$ Pediatricians $\cdot$ Knowledge

\section{Introduction}

Celiac disease is a chronic enteropathy triggered by gluten ingestion. In genetically susceptible individuals, their immune response to gluten provokes alterations in the small-bowel mucosa characterized by lymphocyte infiltration, crypt hyperplasia, and atrophy of villi [1]. Nevertheless, the complications of celiac disease are not confined to alterations in the gastrointestinal tract and may affect the skin, connective tissue, endocrine system, and central nervous system, and include malignant complications such as lymphoma [1-3].

The prevalence of celiac disease is estimated at $0.5-1 \%$ of the general population $[1,4]$, and it is more common in females $[1,4]$. However, the disease remains to be 
under-diagnosed. Patients may have symptoms, either frequently or rarely, for up to 10 years prior to obtaining a definitive diagnosis. This delay in diagnosing the disease may be attributed to physicians' lack of knowledge in this area [5].

The classic presentation of celiac disease consists of gastrointestinal symptoms; however, some patients may be asymptomatic or have atypical symptoms. In view of this variety of presentations and the poor proportion of diagnosed cases, specialists all agree with the idea of a "celiac iceberg" in which the number of undiagnosed cases is considerably greater than the number of diagnosed cases [1,2].

In accordance with its clinical manifestations, celiac disease is classified as classic, atypical, silent, or latent. Classic celiac disease is characterized by poor bowel absorption and predominantly gastrointestinal symptoms such as chronic diarrhea, abdominal pain, vomiting, asthenia, and weight loss, whereas in atypical celiac disease extraintestinal signs or symptoms such as iron-deficiency anemia, folic acid deficiency, osteoporosis, dermatitis herpetiformis, unexplained hypertransaminasemia, and neurological alterations are also present $[1,2]$. Persistent exposure to gluten leads to a reduction in serum calcium and vitamin D levels, which may contribute to bone remodeling and the development of osteoporosis [6]. Celiacs may have much lower bone mineral density levels compared to non-celiacs, suggesting that this enteropathy contributes towards exacerbating osteoporosis [6].

Another important pathology associated with celiac disease is non-Hodgkin lymphomas, principally T-cell lymphomas primary to the bowel. Although rare, this type of lymphoma has been reported in association with long-term celiac disease and is more often found between the sixth and seventh decades of life [1-3]. In these cases, the lymphoma is believed to be a complication of celiac disease, thereby constituting a malignant disease that may be avoided by eliminating gluten from the diet of genetically susceptible individuals.

Clear symptomatology may be absent even when a typical lesion of the duodenal mucosa is present, and in such cases, celiac disease is classified as silent, which does not mean that these patients will not suffer complications in the future. On the other hand, in the latent form, there are no typical lesions of the duodenal mucosa or symptoms suggestive of the disease; however, autoimmunity associated with genetic predisposition is present $[1,7]$. There is a genetic susceptibility in celiac disease in view of the high concordance between monozygotic twins and by the association of the disease with certain human leukocyte antigens (HLA) class II, particularly HLA-DQ2, found in $95 \%$ of patients, and HLA-DQ8 [1, 2].

Lesions to the bowel mucosa occur through activation of the immune system following exposure to gliadin, the part of gluten that is soluble in alcohol. Tissue transglutaminase
(tTG), the principal autoantigen of celiac disease, converts gliadin peptides into glutamic acid, which binds to the HLA molecules of the antigen-presenting cells and induces the proliferation of gliadin-specific CD4 + T-lymphocyte clones [1]. The serological tests for celiac disease include assessment of $\mathrm{IgA}$ and $\operatorname{IgG}$ anti-gliadin antibodies (IgA-AGA, IgG-AGA), IgA anti-endomysium antibodies (IgA-EmA) and $\operatorname{IgA}$ anti-tissue transglutaminase antibodies (IgA anti-tTG) [5]. The use of AGA at screening or during the follow-up of patients with celiac disease has diminished due to the poor sensitivity and specificity of this antibody (around 50\%) and also to the development of other serological markers [1]. Anti-tTG IgA measurement was established as the first choice for screening patients for celiac disease after its high sensitivity of over $90 \%$ was confirmed in various studies [1, 2, 8]. Nevertheless, immunoglobulin A ( $\operatorname{Ig} \mathrm{A})$ deficiency occurs in 1.7-2.6\% of celiac patients and, when present, IgG anti-tTG investigation is recommended [1]. The most specific serological test is anti-EmA measurement, with specificity close to $100 \%$ [1]. If these tests are positive, a diagnosis of celiac disease should be confirmed by duodenal biopsy.

Biopsy findings should be interpreted according to the criteria defined by Marsh for the classification of a lesion of the duodenal mucosa. In a typical celiac lesion, there is an increase of more than $30 \%$ in the proportion of intraepithelial lymphocytes, crypt hyperplasia, and villous atrophy (Marsh III) [2]. Therefore, celiac disease is a condition that involves systemic manifestations of which general practitioners and specialists should be aware in order to ensure early diagnosis and implementation of appropriate treatment. Once diagnosis is confirmed, the clinician should screen patients for iron and folic acid deficiency, request bone mineral density evaluation, implement calcium supplementation if necessary, and refer these patients to a nutritionist. A lifelong, gluten-free diet is the only way to avoid the morbidity and complications resulting from this disease [9].

According to the American Gastroenterological Society, physicians need to be aware of how to screen for celiac disease in risk groups such as first-degree relatives of celiac patients, individuals with type 1 diabetes mellitus, Turner's syndrome, low stature, iron deficient anemia, autoimmune thyroiditis, Down syndrome, and permanent erosion of dental enamel. Some studies have shown these conditions to be 3-10 times more common in patients with celiac disease [1, 10-12]. Celiac disease remains shrouded in numerous myths resulting from the limited information that was available on the disease in the past, despite the fact that it is now well documented in current medical literature. Many clinicians have a poor understanding of this disease and erroneously believe it to be a rare condition that presents as a syndrome of poor bowel absorption and is restricted to Caucasian populations [5]. 
The objective of the present study was to determine the level of awareness of pediatricians with respect to celiac disease, evaluating their knowledge on aspects such as prevalence, diagnosis, treatment and the pathologies associated with this disease.

\section{Methods}

A descriptive, cross-sectional study was conducted in a convenience sample composed of pediatricians participating in the Nestlé Pediatrics Course held in the city of Natal, Brazil, in 2008. Physicians were asked to complete a selfapplicable questionnaire covering aspects concerning the prevalence, diagnosis, and treatment of celiac disease and the conditions associated with this disease. All pediatricians who had been asked to answer the questionnaire did so. Only $3.2 \%$ of the pediatricians were pediatric gastroenterologists. The data were analyzed using the SPSS statistical software program, version 14.0 for Windows. Descriptive measures of frequency and dispersion were used. Prior to participation in the study, candidate participants were informed with respect to the objectives of the study and voluntarily agreed to participate by signing an informed consent form.

\section{Results}

A total of 632 pediatricians completed the questionnaire. The majority of respondents $(82.9 \%)$ were female. All the geographical regions of Brazil were represented; however, the greatest proportion of participants in the sample came from the northeast and southeast of the country. More than $65 \%$ of respondents had undergone specialist training in pediatrics and $31.5 \%$ worked in subspecialties of pediatrics. More than $40 \%$ of respondents had worked as pediatricians for more than 25 years, with a mean of $21.1 \pm$ 10.06 years. The largest proportion of respondents worked in outpatient departments or in infirmaries. Working simultaneously in both the private and public healthcare sectors was more common than working in just one of these areas alone (Table 1).

With respect to the prevalence of celiac disease, only $4.3 \%$ of respondents were able to answer this question. Approximately $91 \%$ of doctors stated that they had provided care to fewer than ten patients with celiac disease and only $9 \%$ had provided care for more than ten patients with this pathology. Regarding the clinical manifestations of the disease, more than $90 \%$ of respondents recognized chronic diarrhea and abdominal distention as symptoms of celiac disease; however, fewer than $70 \%$ were able to identify recurrent abdominal pain as a clinical manifestation of
Table 1 Demographic and professional characteristics of the pediatricians interviewed

\begin{tabular}{lrr}
\hline Characteristic & $n$ (total) & $(\%)$ \\
\hline Female & $496(597)$ & 82.9 \\
Region & & \\
Northeast & $335(573)$ & 59.2 \\
North & $30(573)$ & 5.3 \\
Southeast & $120(573)$ & 21.2 \\
Midwest & $56(573)$ & 9.9 \\
South & $25(573)$ & 4.4 \\
Medical residency in pediatrics & $414(596)$ & 65.5 \\
Time working in pediatrics & & \\
$\leq 5$ years & $63(579)$ & 10.9 \\
$>5$ years and $\leq 15$ years & $99(579)$ & 17.1 \\
$>16$ years and $\leq 25$ years & $169(579)$ & 29.2 \\
$>25$ years & $248(579)$ & 42.8 \\
Works in a subspecialty of pediatrics & $193(613)$ & 31.5 \\
Place of work & & \\
Outpatient service & $545(624)$ & 87.3 \\
Infirmary & $151(624)$ & 24.2 \\
Emergency department & $279(624)$ & 44.7 \\
Pediatric intensive care unit & $39(624)$ & 6.3 \\
Neonatal intensive care unit & $104(624)$ & 16.7 \\
Sector in which participant is employed & & \\
Only in the public sector & $177(588)$ & 30.1 \\
Only in the private sector & $99(588)$ & 16.8 \\
Both public and private sectors & $312(588)$ & 53.1 \\
\hline
\end{tabular}

Table 2 Response frequency on the manifestations of celiac disease

\begin{tabular}{lll}
\hline Clinical manifestations & $n$ (total) & $(\%)$ \\
\hline Chronic diarrhea & $576(632)$ & 91.1 \\
Abdominal distention & $575(632)$ & 91 \\
Low weight & $529(632)$ & 83.7 \\
Short stature & $489(632)$ & 77.4 \\
Anemia & $465(632)$ & 73.6 \\
Recurrent abdominal pain & $425(632)$ & 67.2 \\
Irritability & $318(613)$ & 51.9 \\
Vomiting & $157(632)$ & 24.8 \\
Constipation & $154(632)$ & 24.4 \\
Asymptomatic & $140(632)$ & 22.2 \\
\hline
\end{tabular}

the disease. Approximately 22\% replied that celiac disease may be asymptomatic (Table 2).

Of the pediatricians, $57 \%$ stated that antigliadin antibody measurement represents the best screening tool for celiac disease. Only a quarter of the respondents replied that the best screening tool is anti-tissue transglutaminase antibody measurement. When asked which method should be used to obtain a definitive diagnosis and approximately 
Table 3 Distribution of responses regarding the best screening test and the best confirmatory test for celiac disease

\begin{tabular}{lrr}
\hline & $n$ (total) & $\%$ \\
\hline Best screening test for celiac disease & & \\
Anti-gliadin antibody & $364(632)$ & 57.6 \\
Anti-endomysium antibody & $269(632)$ & 42.6 \\
Anti-tissue transglutaminase antibody & $161(632)$ & 25.5 \\
Fecal fat assessment & $141(632)$ & 22.3 \\
HLA DQ2 and DQ8 & $29(632)$ & 4.6 \\
Bowel biopsy & $4(632)$ & 0.6 \\
None & $15(632)$ & 2.4 \\
Did not know & $92(632)$ & 14.6 \\
Confirmatory test for celiac disease & & \\
Bowel biopsy & $425(632)$ & 67.2 \\
Anti-gliadin antibody & $106(632)$ & 16.8 \\
Anti-endomysium antibody & $74(632)$ & 11.7 \\
Anti-tissue transglutaminase antibody & $60(632)$ & 9.5 \\
HLA DQ2 and DQ8 & $33(632)$ & 5.2 \\
None & $6(632)$ & 0.9 \\
Did not know & $113(632)$ & 17.9 \\
\hline
\end{tabular}

two-thirds replied that bowel biopsy would be the most indicated method (Table 3).

The pathologies and conditions associated with celiac disease were identified by fewer than $50 \%$ of respondents. The most frequently mentioned factor associated with the condition was the existence of first-degree relatives with celiac disease. Some diseases strongly linked to celiac disease were less frequently mentioned by the pediatricians, such as type 1 diabetes mellitus (17.1\%), autoimmune thyroiditis $(13.3 \%)$, and dermatitis herpetiformis (10.6\%). The pathology least likely to be identified by the pediatricians as being associated with celiac disease was neurological manifestations (Table 4).

Exclusion of gluten from the patient's diet was mentioned as the treatment for celiac disease by $86.4 \%$ of the pediatricians, $77.6 \%$ of who stated that the treatment should be continued throughout the patient's life.

The pediatricians were questioned regarding their need for further information on celiac disease and $99.1 \%$ replied that they would indeed benefit from access to further information.

\section{Discussion}

Celiac disease affects $0.5-1 \%$ of the population worldwide [1]. It is noteworthy that even with a mean experience of 21 years in the profession, over $90 \%$ of the pediatricians interviewed replied that they had provided care to fewer than ten patients with celiac disease. Moreover, most of
Table 4 Frequency of responses regarding pathologies or conditions associated with celiac disease

\begin{tabular}{llr}
\hline Associated conditions or pathologies & $n$ (total) & $\%$ \\
\hline First-degree relatives with celiac disease & $284(632)$ & 44.9 \\
Delayed puberty & $241(632)$ & 38.1 \\
Osteoporosis & $230(632)$ & 36.4 \\
Immunoglobulin A deficiency & $205(632)$ & 32.4 \\
Hypoplasia of dental enamel & $148(632)$ & 23.4 \\
Neoplasia & $125(632)$ & 19.8 \\
Aphthae & $122(632)$ & 19.3 \\
Type 1 diabetes mellitus & $105(613)$ & 17.1 \\
Infertility & $99(632)$ & 15.7 \\
Autoimmune thyroiditis & $84(632)$ & 13.3 \\
Dermatitis herpetiformis & $67(632)$ & 10.6 \\
Down syndrome & $49(632)$ & 7.8 \\
Peripheral neuropathy & $41(632)$ & 6.5 \\
Ataxia & $14(632)$ & 2.2 \\
Epilepsy & $10(632)$ & 1.6 \\
\hline
\end{tabular}

these physicians were working in outpatient departments or in infirmaries where the possibility of identifying patients with celiac disease is theoretically greater than that of professionals working in intensive care units, for example. This finding may reflect these pediatricians' lack of knowledge with respect to the disease and may confirm the suspected under-diagnosis of the condition.

The classic manifestations of celiac disease such as abdominal distention, chronic diarrhea and recurrent abdominal pain were recognized by the majority of pediatricians. However, only $22 \%$ of respondents stated that individuals with celiac disease could be asymptomatic. Despite recognizing the classic signs and symptoms of celiac disease [3], the majority of pediatricians were unaware of which screening test to request. Antigliadin antibody measurement has been used as a screening test in the past; however, its poorer sensitivity and specificity compared to anti-tissue transglutaminase antibody measurement rendered the former a test that is seldom used today. The latest guidelines recommend anti-tissue transglutaminase antibody measurement as the best screening test for patients in whom celiac disease is suspected $[1,2]$.

In recent years, many studies have been published on the atypical forms of celiac disease and its associated pathologies. The idea of a "celiac iceberg" has been widely debated in the medical literature [1-3, 8] and individuals with pathologies such as type 1 diabetes mellitus [12], autoimmune thyroiditis [10], low stature [11], ataxia, peripheral neuropathy and epilepsy [13], and individuals with first-degree relatives affected by celiac disease [7], among others, are considered to represent risk groups for this disease. Of the pediatricians interviewed, fewer than 
$50 \%$ were able to name an associated pathology or condition. Even in the case of factors that are well known to be associated with celiac disease such as type 1 diabetes mellitus, having first-degree relatives affected by the condition, and dermatitis herpetiformis, the percentage of pediatricians able to identify these conditions as being associated with celiac disease was very low. In this study sample, neurological manifestations were found to constitute the least well-known disorder associated with celiac disease. In other words, many patients with these pathologies or conditions are not being investigated for celiac disease, meaning that many children remain undiagnosed and consequently untreated.

With respect to treatment, the pediatricians interviewed were aware that gluten had to be excluded from the diet of patients with celiac disease; however, approximately a quarter failed to recognize that this treatment must be life-long.

Zipser et al. [14] showed that only $11 \%$ of adult celiac disease patients have been diagnosed by primary care physicians in United States. Basic information about celiac disease, like presence of diarrhea (90\%), has been well reported, but some conditions like irritability, associations with diabetes mellitus, anemia and osteoporosis, and the role of anti-endomysial test were reported by less than $50 \%$ of physicians. In Europe, the awareness of celiac disease seems to be better. Steens et al. [15] reported that $99 \%$ of Dutch pediatricians used some "celiac antibody" to diagnosis celiac disease and affirm that there was a significant increase in typical and not-typical celiac disease diagnosis (atypical, latent) between 1993 and 2000. However, Auricchio et al. [16] studded 54 Italian centers for the diagnosis of CD, and showed that $52.5 \%$ of those centers still use antigliadin for celiac disease diagnosis, up to $67.5 \%$ consider an infiltrative lesion (Marsh I) and 15\% a normal mucosa and presence of positive serology consistent with celiac disease.

It is important to emphasize that this study does not reflect the knowledge of all pediatricians in Brazil with respect to celiac disease, since this was a convenience study and therefore cannot be considered representative of the population as a whole. However, the fact that this sample population was recruited at a scientific meeting suggests that the pediatricians interviewed in the study may be more up-to-date scientifically than the majority of physicians. If this is indeed the case, the current status concerning the level of knowledge of physicians in general with respect to celiac disease may be even worse than that identified in this study.

\section{Conclusions}

Although celiac disease exists worldwide, with significant morbidity and various associated conditions and pathologies, the knowledge of pediatricians with respect to this disease remains poor. The classic symptoms such as chronic diarrhea and abdominal pain and treatment through implementation of a gluten-free diet appear to represent the most commonly known facts of the disease among pediatricians. Nevertheless, this information is superficial and the majority of pediatricians remain unaware of relevant issues concerning atypical manifestations, diagnosis, associated conditions and pathologies, and the duration of treatment.

The need for relevant information on celiac disease is fundamental, and is recognized by the pediatricians themselves. Actions involving continued education and placing a greater emphasis on providing information on celiac disease in undergraduate and postgraduate medical training are fundamental, since this disease involves diverse systemic manifestations that are not confined to gastroenterology.

\section{References}

1. Rostom A, Murray JA, Kagnoff MF. American Gastroenterological Association (AGA) Institute technical review on the diagnosis and management of celiac disease. Gastroenterology. 2006;131:1981-2002.

2. Catassi C, Fasano A. Celiac disease. Curr Opin Gastroenterol. 2008;24:687-691.

3. Fasano A. Clinical presentation of celiac disease in the pediatric population. Gastroenterology. 2005;128:S68-S73.

4. Melo SB, Fernandes MI, Peres LC, Troncon LE, Galvao LC. Prevalence and demographic characteristics of celiac disease among blood donors in Ribeirao Preto, State of Sao Paulo, Brazil. Dig Dis Sci. 2006;51:1020-1025.

5. Zipser RD, Farid M, Baisch D, Patel B, Patel D. Physician awareness of celiac disease: a need for further education. J Gen Intern Med. 2005;20:644-646.

6. Sugai E, Chernavsky A, Pedreira S, et al. Bone-specific antibodies in sera from patients with celiac disease: characterization and implications in osteoporosis. J Clin Immunol. 2002;22: $353-362$.

7. Goldberg D, Kryszak D, Fasano A, Green PH. Screening for celiac disease in family members: is follow-up testing necessary? Dig Dis Sci. 2007;52:1082-1086.

8. Collin P, Huhtala H, Virta L, Kekkonen L, Reunala T. Diagnosis of celiac disease in clinical practice: physician's alertness to the condition essential. J Clin Gastroenterol. 2007;41:152-156.

9. Hopman EG, le Cessie S, von Blomberg BM, Mearin ML. Nutritional management of the gluten-free diet in young people with celiac disease in The Netherlands. J Pediatr Gastroenterol Nutr. 2006;43:102-108.

10. da Silva Kotze LM, Nisihara RM, da Rosa Utiyama SR, Piovezan GC, Kotze LR. Thyroid disorders in Brazilian patients with celiac disease. J Clin Gastroenterol. 2006;40:33-36.

11. Queiroz MS, Nery M, Cancado EL, Gianella-Neto D, Liberman B. Prevalence of celiac disease in Brazilian children of short stature. Braz. J Med Biol Res. 2004;37:55-60.

12. Tanure MG, Silva IN, Bahia M, Penna FJ. Prevalence of celiac disease in Brazilian children with type 1 diabetes mellitus. $J$ Pediatr Gastroenterol Nutr. 2006;42:155-159.

13. Siqueira Neto JI, Costa AC, Magalhaes FG, Silva GS. Neurological manifestations of celiac disease. Arq Neuropsiquiatr. 2004;62:969-972. 
14. Zipser RD, Farid M, Baisch D, Patel B, Patel D. Physician awareness of celiac disease: a need for further education. $J$ Gen Intern Med. 2005;20(7):644-646.

15. Steens RF, Csizmadia CG, George EK, Ninaber MK, Hira Sing RA, Mearin ML. A national prospective study on childhood celiac disease in the Netherlands 1993-2000: an increasing recognition and a changing clinical picture. J Pediatr. 2005;147(2): 239-243.
16. Auricchio R, Granata V, Borrelli M, Troncone R. Italian Society for Pediatric Gastroenterology Hepatology and Nutrition (SIGENP). Italian paediatricians' approach to coeliac disease diagnosis. J Pediatr Gastroenterol Nutr. 2009;49(3):374-376. 